\title{
Strategy-proof allocation mechanisms for pure public goods economies when preferences are monotonic ${ }^{\star}$
}

\section{Diego Moreno}

Departamento de Economía, Universidad Carlos III de Madrid, E-28903 Getafe, SPAIN (e-mail:dmoreno@eco.uc3m.es)

Received: August 14, 1995; revised version: September 25, 1997

Summary. A fundamental problem in public finance is that of allocating a given budget to financing the provision of public goods (education, transportation, police, etc.). In this paper it is established that when admissible preferences are those representable by continuous and increasing utility functions, then strategy-proof allocation mechanisms whose (undominated) range contains three or more outcomes are dictatorial on the set of profiles of strictly increasing utility functions, a dense subset of the domain in the topologies commonly used in this context. If admissible utility functions are further restricted to be strictly increasing, or if mechanisms are required to be non-wasteful, then strategy-profness leads to (full) dictatorship.

JEL Classification Numbers: D60, D70, H40.

\section{Introduction}

A fundamental problem in public finance is that of deciding how to allocate a given (limited) budget to financing the provision of public goods (education, transportation, police, etc.). The purpose of this paper is to characterize the kinds of institutions, or allocation mechanisms, that can be used to make these decisions. It is shown that if the preferences of those individuals affected by the decision are to be taken into consideration, then all allocation mechanisms have very unappealing properties: either they are not compatible

\footnotetext{
^ I wish to thank an anonymous referee for pointing out the presence of an error in a previous draft, and for many helpful suggestions. I have benefitted from discussions with Salvador Barberà and Mark Walker, and from comments by Ezra Einy, José Luis Ferreira, Monique Florenzano, Carlos Hervés, Larry Kranich, Javier Ruiz-Castillo, and Lin Zhou. Financial support from the Ministerio de Asuntos Sociales through funds administered by the Cátedra Gumersindo de Azcárate, and from DGICYT grant PB93-2030 are gratefully acknowledged.
} 
with individuals' incentives, or they select outcomes on the basis of the preferences of a single individual.

The revelation principle has established that the search for incentive compatible allocation mechanisms can be restricted to those for which each individual's strategy space is the set of his possible preferences. An allocation mechanism in this class is a mapping which associates a feasible outcome with each profile of reported preferences. Since individuals' preferences are private information, it is conceivable that an individual might attempt to manipulate a mechanism by reporting false preferences. A mechanism is strategy-proof if each individual is best off reporting his true preferences, whatever preferences the other individuals report. Hence strategy-proof mechanisms are not subject to manipulation by any individual.

Whether or not one can design strategy-proof allocation mechanisms that perform well in other respects depends on the domain of preferences on which decisions are to be made, as well as on the set of feasible outcomes. Gibbard [3] and Satterthwaite [10] have shown that when all preferences are admissible, then all strategy-proof decision mechanisms whose range contains three or more outcomes are dictatorial; i.e., they select outcomes that maximize the welfare of a single individual (the dictator). Barberà and Peleg [2] have established that this result remains valid even if admissible preferences are restricted to be continuous. (When preferences are known to be convex as well as continuous, strategy-proof mechanisms with a one dimensional range were characterized by Moulin [8] as median voter type, among which there are nondictatorial ones; this characterization has been generalized recently by Barberà and Jackson [1]. Zhou [11] has shown that strategy-proof decision mechanisms whose range contains a two dimensional set are dictatorial.) In most allocation problems associated with public goods provision, however, individuals' preferences are known to be monotonic on the set of feasible outcomes. Hence these allocation problems are not within the scope of any of the above mentioned results. (Satiated preferences play a fundamental role in the proofs of these results.)

In this paper it is investigated which allocation mechanisms are strategyproof for a domain of preferences typically associated with public goods provision; namely, those representable by continuous and increasing utility functions. The results presented here establish that conclusions similar to those obtained by Gibbard, Satterthwaite, and Barberà-Peleg still hold when admissible preferences are further restricted to be monotonic: It is shown that strategy-proof allocation mechanisms whose domain is the set of all the profiles of continuous and increasing utility functions, and whose (undominated) range contains three or more outcomes are dictatorial in a sense weaker than that commonly used in this context; specifically, these mechanisms are dictatorial on the set of profiles of strictly increasing utility functions, rather than on the entire domain. (The cardinality condition imposed on the mechanism's range implies that at least two public goods are provided.) 
Unfortunately, although this conclusion is weaker than those obtained when satiated preferences are admissible, it is nevertheless very negative: The subdomain where these mechanisms are shown to be dictatorial (the set of profiles of strictly increasing utility functions) is a dense subset of the domain in the topologies commonly used in these contexts. In fact, it is shown that if a mechanism is non-wasteful (or efficient) as well as strategy-proof, then it must be (fully) dictatorial. It is also shown that when the set of admissible utility functions is the set of all the continuous and strictly increasing utility functions, then only (fully) dictatorial mechanisms are strategy-proof.

\section{The model and the results}

The set of individuals is $N=\{1, \ldots, n\}$, where $n \geq 1$. For simplicity, each individual consumption set is taken to be $\Re_{+}^{m}$. The set of feasible outcomes is denoted by $X$, which is assumed to be a compact subset of $\Re_{+}^{m}$. Preferences are represented by utility functions (i.e., real-valued functions on $\Re_{+}^{m}$ ). As individuals' utility functions might be known a priori to have certain properties, let $U$ denote the set of admissible utility functions. For $\mathbf{u} \in U^{n}$ and $S \subset N, \mathbf{u}_{S}$ is the profile obtained from $\mathbf{u}$ by deleting the utility functions of the members of $S$.

An allocation mechanism (or simply a mechanism) is a mapping $f: U^{n} \rightarrow X$. A mechanism $f$ is manipulable by Individual $i$ at $\mathbf{u}=$ $\left(\mathbf{u}_{i}, u_{i}\right) \in U^{n}$ if there is $u^{\prime} \in U$ such that $u_{i}\left(f\left(\mathbf{u}_{i}, u^{\prime}\right)\right)>u_{i}(f(\mathbf{u}))$. A mechanism is strategy-proof if it is not manipulable by any $i \in N$ at any $\mathbf{u} \in U^{n}$. A mechanism $f$ is dictatorial on $\Omega \subset U^{n}$ if there is an individual $i \in N$ such that for each $\mathbf{u} \in \Omega, f(\mathbf{u})$ maximizes $u_{i}$ on $f(\Omega)$ (then Individual $i$ is referred to as a dictator for $f$ on $\Omega$ ). A mechanism $f$ is dictatorial if it is dictatorial on $U^{n}$ (and a dictator for $f$ on $U^{n}$ is simply referred to as a dictator).

Strategy-proof mechanisms are those for which an individual is always best off reporting a utility function representing his true preferences. Thus, when preferences are private information and individuals' prior beliefs about the preferences of the other individuals are unknown, as is often the case, strategy-proofness implies that "truthful revelation" is an "equilibrium." A dictatorial mechanism always selects an outcome from the set of maximizers on the mechanism's range of the dictator's reported utility function. Dictatorial mechanisms are therefore very unsatisfactory as whenever there is a conflict between the dictator's and the other individuals' preferences, the dictator's preferences prevail.

There is a large literature investigating the properties of strategy-proof mechanisms. This literature has generally found that, in certain domains, strategy-proof mechanisms have very unappealing properties. Of course, there are trivial mechanisms that are strategy-proof. For example, any constant mechanism is strategy-proof. Interesting mechanisms, however, are those that are "responsive" to individuals' preferences (as measured, for example, by the size of the mechanism's range). It is therefore important to determine the properties of strategy-proof mechanisms. When satiated 
preferences are admissible, Gibbard [3], Satterthwaite [10], and Barberà and Peleg [2] have established that only dictatorial mechanisms are strategyproof. Barberà and Peleg establish this result for mechanisms whose domain contain all the continuous utility functions and whose range contain three or more outcomes. (When only two outcomes are selected "majority rule" is a strategy-proof and nondictatorial mechanism.)

These results, however, have no implications for most allocation problems associated with public goods provision, as it is usually assumed that individuals' preferences are monotonic; i.e., only increasing utility functions are admissible. A utility function $u$ is increasing if for each $a, b \in \Re_{+}^{m}, a>b$ (i.e., $a_{k} \geq b_{k}$ for $k=1, \ldots, m$ and $a \neq b$ ) implies $u(a) \geq u(b)$, and $a \gg b$ (i.e., $a_{k}>b_{k}$ for $\left.k=1, \ldots, m\right)$ implies $u(a)>u(b)$; it is strictly increasing if $a>b$ implies $u(a)>u(b)$.

Given a set $A \subset \Re^{m}$, write $\widehat{A}$ for the set $\left\{a \in A \mid \nexists a^{\prime} \in A: a^{\prime}>a\right\}$. Thus, if $A \subset \Re^{2}$, then $\widehat{A}$ is the northeast boundary of $A$. Since the domain considered here contains profiles of utility functions that are increasing, then the "relevant" set of outcomes in the range of a mechanism $f$ is the set $f\left(\widehat{U^{n}}\right)$. (Henceforth this set is referred to as the undominated range of a mechanism). Thus, a cardinality condition as that imposed in the Barberà-Peleg Theorem will be imposed on the undominated range of a mechanism, rather than on its entire range. Denote by $\dot{U}$ the set of admissible utility functions that are strictly increasing.

Theorem 1 below establishes a result analogous to the Barberà-Peleg Theorem when admissible utility functions are restricted to be increasing. The proof of Theorem 1, and propositions 1 and 2 below, are given in Section 3 .

Theorem 1. Let $U$ be the set of all the continuous increasing utility functions, and let $f: U^{n} \rightarrow X$ be a strategy-proof mechanism such that $\widehat{f\left(U^{n}\right)}$ contains three or more outcomes. Then $f$ is dictatorial on $\dot{U}^{n}$. Moreover, if Individual $i$ is the dictator for $f$ on $\dot{U}^{n}$, then for each $\mathbf{u} \in \dot{U}^{n}, f(\mathbf{u})$ maximizes $u_{i}$ on $f\left(U^{n}\right)$.

Thus, mechanisms satisfying the assumptions of Theorem 1 are dictatorial on an important subdomain; namely, the set $\dot{U}^{n}$ of profiles of strictly increasing utility functions. (Note that the set $\dot{U}$ is a dense subset of $U$ in the most commonly used topologies; e.g., the compact-open topology, or the topology of closed convergence, i.e., the topology on the set of equivalence classes of $U$ induced by the pseudometric $d$ given for $u^{\prime}, u^{\prime \prime} \in U$ by $d\left(u^{\prime}, u^{\prime \prime}\right)=\delta\left(G\left(u^{\prime}\right), G\left(u^{\prime \prime}\right)\right)$, where $\delta$ is the Hausdorff distance, and for $u \in U, G(u)$ is the set $\left\{(x, y) \in \Re_{+}^{m} \times \Re_{+}^{m} \mid u(x) \geq u(y)\right\}$-see Hildenbrand [4].) Moreover, on this subdomain mechanisms must select the dictator's most preferred outcome on the entire range. Also note that the condition imposed in Theorem 1 on the cardinality of the set $\widehat{f\left(U^{n}\right)}$ effectively requires that at least two public goods be provided. (If $X \subset \Re$, then for any $A \subset X, \widehat{A}$ is a singleton.) 
The conclusion of Theorem 1 is weaker than that obtained by BarberàPeleg, but as Example 1 shows a dictator on $\dot{U}^{n}$ need not be a full dictator.

Example 1. There are three public goods, and the set of feasible outcomes is $X=\left\{x \in \Re_{+}^{3} \mid x_{1}+x_{2}+x_{3} \leq 1\right\}$. Let $f: U^{n} \rightarrow X$ be given for each $\mathbf{u} \in U^{n}$ by $f(\mathbf{u})=(0,0,0)$ if $u_{2}=\bar{u}_{2}$, where $\bar{u}_{2}(x)=x_{1} x_{2} x_{3}$, for $x \in \Re_{+}^{3}$; otherwise let $f(\mathbf{u})$ be some arbitrary maximizer of $u_{1}$ on $\{(1,0,0),(0,1,0),(0,0,1)\}$.

This mechanism is strategy-proof: Clearly Individual 1 cannot manipulate as he gets one of his best outcomes (on the mechanism's range) whenever Individual 2 does not declare $\bar{u}_{2}$, and otherwise the utility function he reports does not affect the outcome. Individual 2 cannot manipulate either: If his preferences are represented by $\bar{u}_{2}$, then he is indifferent between the outcome the mechanism selects when he reports $\bar{u}_{2}$, and all the other outcomes in the range of the mechanism; and if the mechanism selects any other outcome, he can only induce $(0,0,0)$, which can never make him better off. Individuals $3, \ldots, n$ cannot manipulate either as the utility functions they report do not affect the outcome. Moreover, the set $\widehat{f\left(U^{n}\right)}=\widehat{X}$ satisfies the cardinality condition imposed on Theorem 1. Thus, if $U$ is the set of all the continuous increasing utility functions, then $f$ satisfies the assumptions of Theorem 1. Of course, this mechanism is dictatorial on $\dot{U}^{n}$, but it is not (fully) dictatorial.

Under this mechanism Individual 2 never benefits when Individual 1 (the dictator for $f$ on $\dot{U}^{n}$ ) does not get his most preferred outcome. In fact, as Lemma 11 of Section 3 shows, under the assumptions of Theorem 1 the maximizer of the dictator's (for $f$ on $\dot{U}^{n}$ ) reported utility function imposes an upper bound to the bundle the mechanism selects. Thus, every mechanism $f$ which is dictatorial on $\dot{U}^{n}$, but which is not fully dictatorial, is wasteful (i.e., it does not always exhaust the existing resources); moreover, it is also inefficient as whenever the dictator for $f$ on $\dot{U}^{n}$ does not get one of his most preferred outcomes, it is possible to make him better off without making anybody else worse off.

Proposition 1 below establishes that if a mechanism satisfying the assumptions of Theorem 1 is non-wasteful (i.e., is such that $f\left(U^{n}\right) \subset \widehat{X}$ ), then it is (fully) dictatorial. Hence, wastefulness is a property common to all the mechanisms satisfying the assumptions of Theorem 1 that are not (fully) dictatorial. An argument similar to the one given in the proof of Proposition 1 establishes that if a mechanism satisfying the assumptions of Theorem 1 is efficient, then it is dictatorial. (A mechanism $f$ is efficient if for each $\mathbf{u} \in U^{n}$, $f(\mathbf{u})$ is Pareto optimal with respect to $\mathbf{u}$. Since admissible utility functions need not be strictly increasing, wastefulness does not imply inefficiency.)

Proposition 1. Let $U$ be the set of all the continuous increasing utility functions, and let $f: U^{n} \rightarrow X$ be a strategy-proof and non-wasteful mechanism such that $\widehat{f\left(U^{n}\right)}$ contains three or more outcomes. Then $f$ is dictatorial.

Also it is worth noticing that the mechanism defined in Example 1 is discontinuous. One can show that if a mechanism satisfying the assumptions 
of Theorem 1 is continuous (in a very weak sense), then it is (fully) dictatorial (see Moreno [7]).

Theorem 1 rises the question whether one might be able to design mechanisms that perform better when individuals are known to have preferences representable by continuous and strictly increasing utility functions. Proposition 2, however, provides a negative answer to this question. Given an arbitrary set $A \subset \Re^{m}$, denote by $\bar{A}$ its closure.

Proposition 2. Let $\dot{U}$ be the set of all the continuous strictly increasing utility functions, and let $f: \dot{U}^{n} \rightarrow X$ be a strategy-proof mechanism such that $\widehat{f\left(\dot{U}^{n}\right)}$ contains three or more outcomes. Then $f$ is dictatorial.

Note that the cardinality condition is imposed on the of the undominated closure of the range of $f$, rather than the undominated range itself. It is easy to show that whenever $f\left(\dot{U}^{n}\right)$ contains three or more outcomes, then $\widehat{f\left(\dot{U}^{n}\right)}$ also does.

Finally, it is worth pointing out that when admissible utility functions are further restricted to be concave, the conclusion of Theorem 1 no longer holds; as shown in Example 2 below, in this case there are strategy-proof and non-dictatorial mechanisms. Moreover, the mechanism in Example 2 has a two-dimensional range; hence the analog of Zhou's Theorem does not hold when admissible utility functions are further restricted to be increasing.

Example 2. There are two public goods, and the set of feasible outcomes is $X=\left\{\left(x_{1}, x_{2}\right) \in \Re_{+}^{2} \mid x_{1}^{2}+x_{2}^{2} \leq 1\right\}$. Let $U$ be the set of increasing and concave utility functions. For each $u \in U$, let $m(u)=\left(m_{1}(u), m_{2}(u)\right)$ denote the maximizer of $u$ on $X$. Consider the mechanism $f: U^{n} \rightarrow X$ which for each $\mathbf{u} \in U^{n}$, selects

$$
f(\mathbf{u})=\left(\mu(\mathbf{u}), \sqrt{ } 1-\mu(\mathbf{u})^{2}\right),
$$

where $\mu(\mathbf{u})$ is the "median" of $m_{1}\left(u_{1}\right), \ldots, m_{1}\left(u_{n}\right)$. (If $n$ is even, pick an arbitrary point in $[0,1]$ and calculate the median adding this point.)

The mechanism $f$ described in Example 2 is not dictatorial on $\dot{U}^{n}$. Moreover, its range is the set $\widehat{X}$, a two dimensional set. It is easy to prove that this mechanism is also strategy-proof: let $i \in N$ and $\mathbf{u} \in U^{n}$ be such that $f(\mathbf{u}) \neq m\left(u_{i}\right)$; then either (1) $f_{1}\left(u_{i}^{\prime}, \mathbf{u}{ }_{i}\right) \geq f_{1}(\mathbf{u})>m_{1}\left(u_{i}\right)$ for each $u_{i}^{\prime} \in U$, or (2) $f_{1}\left(u_{i}^{\prime}, \mathbf{u}{ }_{i}\right) \leq f_{1}(\mathbf{u})<m_{1}\left(u_{i}\right)$ for each $u_{i}^{\prime} \in U$; thus, as $u_{i}$ is increasing and concave one has

$$
u_{i}(f(\mathbf{u})) \geq u_{i}\left(f\left(u_{i}^{\prime}, \mathbf{u}{ }_{i}\right)\right),
$$

for each $u_{i}^{\prime} \in U$. Hence $f$ is strategy-proof.

An important open question is whether one can construct interesting strategy-proof mechanism (as the one in Example 2) for this domain when the dimension of the feasible set is greater than two. 


\section{Proofs}

Let $f: U^{n} \rightarrow X$. We assume throughout that $f$ is a strategy-proof mechanism. For $u_{n} \in U$ let the mechanism $f^{u_{n}}: U^{n}{ }^{1} \rightarrow X$ be given, for each $\mathbf{u}_{n} \in U^{n}{ }^{1}$, by $f^{u_{n}}\left(\mathbf{u}_{n}\right)=f\left(\mathbf{u}_{n}, u_{n}\right)$. Note that each $f^{u_{n}}$ is also a strategyproof mechanism. For each $u_{n} \in U$, write $O\left(u_{n}\right)=f^{u_{n}}\left(U^{n}{ }^{1}\right) \cap f\left(\widehat{\left.U^{n}\right)}\right.$. Thus, the correspondence $O$ provides the "options" (i.e., outcomes in the undominated range) that are attainable by individuals $1, \ldots, n-1$, given the utility function reported by Individual $n$.

The proof of Theorem 1 proceeds along the lines of the proof of the Barberà-Peleg Theorem. As in their proof, the correspondence $O$ plays a fundamental role. The strategy of the proof is to show that this correspondence is constant on a certain subdomain, and furthermore that its value is either a singleton (Individual $n$ 's most preferred outcome) or it is the entire undominated range of $f$. Hence either Individual $n$ is a dictator on this subdomain, or the utility function he reports does not influence the outcome. It is straightforward to show by induction on the number of individuals that this implies that $f$ is a dictatorial mechanism on this subdomain. With this result at hand, one can easily establish Theorem 1 .

There are two basic differences between the proof of B-P's Theorem and the proof of Theorem 1. First, option sets as defined here contain only undominated outcomes, whereas in the proof of B-P's Theorem options sets are the sets $f^{u_{n}}\left(U^{n}{ }^{1}\right)$. Lemma 4 below, however, tells us that whenever all individuals report a strictly increasing utility function, then the outcome belongs to the undominated range (i.e., $f\left(\dot{U}^{n}\right) \subset f\left(\widehat{U^{n}}\right)$ ); this allows one to show that in this subdomain the correspondence $O$ (as defined here) has the property mentioned above: it is constant, and it is either a singleton or it is the entire set $f\left(\widehat{\left.U^{n}\right)}\right.$ (lemmas 8 and 9). Second, when satiated preferences are admissible, option sets are shown to be closed; when only monotonic preferences are admissible, however, option sets need not be closed, although they are shown to be closed relative to $f\left(\widehat{U^{n}}\right)$ (Lemma 6). It turns out that in the construction of the proof (specifically, in the proofs of lemmas 8 and 9) it is only required that one be able to separate out any point in the undominated range which is not in a given option set from the option set itself (i.e., that no point $x \in f\left(\widehat{U^{n}}\right) \backslash O\left(u_{n}\right)$ is in the closure of $\left.O\left(u_{n}\right)\right)$.

Before proving Theorem 1, a number of preliminary results are established. Lemma 1 establishes that strategy-proof mechanisms satisfy a modified version of the strong positive association property. Its proof is omitted (see Lemma 4.8 in Barberà and Peleg [2]).

Lemma 1 (MSPAP) For each $\mathbf{u}=\left(u_{1}, \ldots, u_{n}\right) \in U^{n}, u \in U$ and $i \in N$ such that for every $x \in f\left(U^{n}\right) \backslash\{f(\mathbf{u})\}, u(x) \geq u(f(\mathbf{u}))$ implies $u_{i}(x)>u_{i}(f(\mathbf{u}))$, one has $f\left(\mathbf{u}_{i}, u\right)=f(\mathbf{u})$.

Lemma 2 establishes a standard unanimity property of strategy-proof mechanisms. 
Lemma 2. (Unanimity) For each $u \in U, f(u, \ldots, u)$ maximizes $u$ on $f\left(U^{n}\right)$.

Proof. Let $u \in U$ and $x \in f\left(U^{n}\right)$, and let $\mathbf{u} \in U^{n}$ be such that $f(\mathbf{u})=x$. Successive applications of strategy-proofness imply

$$
u(f(u, \ldots, u)) \geq u\left(f\left(u, \ldots, u, u_{n}\right)\right) \geq \cdots \geq u\left(f\left(u, \mathbf{u}_{-1}\right)\right) \geq u(f(\mathbf{u}))=u(x),
$$

which establishes the lemma.

Write $U^{*}$ for the set containing the utility functions in $U$ with a unique maximizer on $f\left(U^{n}\right)$. Note that the maximizer of each $u \in U^{*}$ on $f\left(U^{n}\right)$, denoted by $m(u)$, is a member of $\overrightarrow{f\left(U^{n}\right)}$. Lemma 3 is a direct implication of Lemma 2: it establishes that whenever Individual $n$ claims a utility function $u_{n}$ in the set $U^{*}$, then $m\left(u_{n}\right)$ is a member of the set $O\left(u_{n}\right)$.

Lemma 3. For each $u_{n} \in U^{*}, m\left(u_{n}\right) \in O\left(u_{n}\right)$.

In the remaining of the proof, assume that $U$ is the set of all the continuous increasing utility functions. Lemma 4 establishes, for the domain under study, that a strategy-proof mechanism must select an outcome in $\widehat{f\left(U^{n}\right)}$ whenever all individuals report a strictly increasing utility function. Lemma 4 therefore implies that for each $\mathbf{u} \in \dot{U}^{n}$, one has $f(\mathbf{u})=f^{u_{n}}\left(\mathbf{u}_{-n}\right) \in O\left(u_{n}\right)$.

Lemma 4. For each $\mathbf{u} \in \dot{U}^{n}, f(\mathbf{u}) \in \widehat{f\left(U^{n}\right)}$.

Proof. Suppose by way of contradiction that there is $\mathbf{u}=\left(u_{1}, \ldots, u_{n}\right) \in \dot{U}^{n}$ such that $f(\mathbf{u}) \notin \widehat{f\left(U^{n}\right)}$. Write $f(\mathbf{u})=\bar{x}$, and choose $\alpha \in \Re^{n}$ such that $u_{1}(\bar{x})+\alpha_{1}=u_{2}(\bar{x})+\alpha_{2}=\ldots=u_{n}(\bar{x})+\alpha_{n}$. Consider the utility function $u$ given for each $x \in \Re_{+}^{m}$ by

$$
u(x)=\min \left\{u_{1}(x)+\alpha_{1}, \ldots, u_{n}(x)+\alpha_{n}\right\} .
$$

Hence $u \in \dot{U}$. Moreover, for each $x \in X \backslash\{\bar{x}\}, \quad u(x) \geq u(\bar{x})$ implies $u_{i}(x) \geq u_{i}(\bar{x})$ for each $i \in N$. Thus, by slightly bending in the direction of the main diagonal $u$ 's indifference curve through $\bar{x}$, one can obtain a utility function $\bar{u} \in \dot{U}$ satisfying for each $x \in X \backslash\{\bar{x}\}$, that $\bar{u}(x) \geq \bar{u}(\bar{x})$ implies $u_{i}(x)>u_{i}(\bar{x})$, for each $i \in N$. MSPAP (Lemma 1) yields

$$
\bar{x}=f(\mathbf{u})=f\left(\bar{u}, u_{2}, \ldots, u_{n}\right)=\ldots=f\left(\bar{u}, \bar{u}, \ldots, \bar{u}, u_{n}\right)=f(\bar{u}, \bar{u}, \ldots, \bar{u}) .
$$

Since $\bar{x} \in f\left(U^{n}\right) \backslash \widehat{f\left(U^{n}\right)}$ there is $\bar{x}^{\prime} \in f\left(U^{n}\right)$ such that $\bar{x}^{\prime}>\bar{x}$, and as $\bar{u}$ is a strictly increasing function, one has $\bar{u}\left(\bar{x}^{\prime}\right)>\bar{u}(\bar{x})=\bar{u}(f(\bar{u}, \bar{u}, \ldots, \bar{u}))$. This contradicts Lemma 2 and establishes Lemma 4.

For each $x \in \Re_{+}^{m}$, let $I(x)$ be the set of indices $k \in\{1, \ldots, m\}$ such that $x_{k}>0$.

Lemma 5. If $x$ is a limit point of $f\left(U^{n}\right)$, then there is $x^{\prime} \in f\left(U^{n}\right)$ such that $x^{\prime} \geq x$. 
Proof. Let $\bar{x}$ be an arbitrary limit point of $f\left(U^{n}\right)$, and suppose by way of contradiction that there is no $\bar{x}^{\prime} \in f\left(U^{n}\right)$ such that $\bar{x}^{\prime} \geq \bar{x}$. Let $\alpha \in \Re^{m}, \alpha \gg 0$, be such that for each $k, k^{\prime} \in I(\bar{x}), \alpha_{k} \bar{x}_{k}=\alpha_{k^{\prime}} \bar{x}_{k^{\prime}}$, and let $u \in U$ be given, for each $x \in \Re^{m}$, by $u(x)=\min \left\{\alpha_{k} x_{k}, k \in I(\bar{x})\right\}$. Note that $u(\bar{x})>u(x)$ for each $x \in f\left(U^{n}\right)$. Write $f(u, \ldots, u)=\tilde{x}$. Hence $u(\bar{x})>u(\tilde{x})$. Moreover, as $\bar{x}$ is a limit point of $f\left(U^{n}\right)$, there is $x \in f\left(U^{n}\right)$ sufficiently close to $\bar{x}$ that $u(x)>u(\tilde{x})$. This contradicts Lemma 2, and establishes Lemma 5.

Lemma 6 establishes that option sets are closed relative to $\widehat{f\left(U^{n}\right)}$.

Lemma 6. For each $u_{n} \in U$, the set $O\left(u_{n}\right)$ is closed relative to $\widehat{f\left(U^{n}\right)}$.

Proof. Let $u_{n} \in U$ arbitrary, and let $\bar{x} \in \widehat{f\left(U^{n}\right)}$ be a limit point of $O\left(u_{n}\right)$. Since $\bar{x}$ is a limit point of $f^{u_{n}}\left(U^{n-1}\right)$, Lemma 5 (applied to $\left.f^{u_{n}}\right)$ implies that there is $\bar{x}^{\prime} \in f^{u_{n}}\left(U^{n-1}\right)$ such that $\bar{x}^{\prime} \geq \bar{x}$; since $\bar{x} \in \widehat{f\left(U^{n}\right)}$, and $f^{u_{n}}\left(U^{n-1}\right) \subset f\left(U^{n}\right)$, one has $\bar{x}^{\prime}=\bar{x}$.

Lemma 7 establishes that if an outcome is not in the undominated range, then there is an outcome in the undominated range which contains more of at least one commodity and no less of any commodity.

Lemma 7. For each $x \in f\left(U^{n}\right) \backslash \widehat{f\left(U^{n}\right)}$, there is $x^{\prime} \in \widehat{f\left(U^{n}\right)}$ such that $x^{\prime}>x$.

Proof. Given $\bar{x} \in f\left(U^{n}\right) \backslash f\left(\widehat{U^{n}}\right)$, let $\bar{x}^{\prime}$ be an arbitrary maximal point of the closure of the set $\left\{x \in f\left(U^{n}\right) \mid x \geq \bar{x}\right.$ \} (note that since $X$ is a compact set, $f\left(U^{n}\right)$ is bounded). If $\bar{x}^{\prime}$ is an isolated point of $f\left(U^{n}\right)$, then $\bar{x}^{\prime} \in \widehat{f\left(U^{n}\right)}$; if $\bar{x}^{\prime}$ is a limit point of $f\left(U^{n}\right)$, then by Lemma 5 there is $\bar{x}^{\prime \prime} \in f\left(U^{n}\right)$ such that $\bar{x}^{\prime \prime} \geq \bar{x}^{\prime}$; but since there is no point $x \in f\left(U^{n}\right)$ such that $x>\bar{x}^{\prime}$, then $\bar{x}^{\prime} \in f\left(U^{n}\right)$, and therefore $\bar{x}^{\prime} \in \widehat{f\left(U^{n}\right)}$.

Denote by $\dot{U}^{*}$ the set $U^{*} \cap \dot{U}$. Lemma 8 establishes that option sets only depend on the maximizer of Individual $n$ 's reported utility function.

Lemma 8. For $u_{n}, u_{n}^{\prime} \in \dot{U}^{*}, m\left(u_{n}\right)=m\left(u_{n}^{\prime}\right)$ implies $O\left(u_{n}\right)=O\left(u_{n}^{\prime}\right)$.

Proof. Suppose, by way of contradiction, that there are $u_{n}, u_{n}^{\prime} \in \dot{U}^{*}$ such that $m\left(u_{n}\right)=m\left(u_{n}^{\prime}\right)=\bar{x}$, and such that there is $y \in O\left(u_{n}\right) \backslash O\left(u_{n}^{\prime}\right)$. Let $\alpha, \alpha^{\prime} \in \Re^{m}$, $\alpha, \alpha^{\prime} \gg 0$, be such that $\alpha_{k} \bar{x}_{k}=\alpha_{k^{\prime}} \bar{x}_{k^{\prime}}$ for $k, k^{\prime} \in I(\bar{x})$, and $\alpha_{k}^{\prime} y_{k}=\alpha_{k^{\prime}}^{\prime} y_{k^{\prime}}$ for $k, k^{\prime} \in I(y)$. Let $u, u^{\prime} \in U^{*}$ be defined, respectively, by $u(x)=$ $\min \left\{\alpha_{k} x_{k}, k \in I(\bar{x})\right\}$, and $u^{\prime}(x)=\min \left\{\alpha_{k}^{\prime} x_{k}, k \in I(y)\right\}$, for each $x \in \Re_{+}^{m}$. Note that $m(u)=\bar{x}$, whereas $m\left(u^{\prime}\right)=y$.

It is now shown that there is $\tilde{x}<y$, such that $\alpha_{k}^{\prime} \tilde{x}_{k}=\alpha_{k^{\prime}}^{\prime} \tilde{x}_{k^{\prime}}$ for $k, k^{\prime} \in I(y)$, and such that there is no $x \in f^{u_{n}^{\prime}}\left(U^{n-1}\right)$ with $x \geq \tilde{x}$ (see Fig. 1).

For each integer $q$, choose $\tilde{x}(q)$ such that $\alpha_{k}^{\prime} \tilde{x}_{k}(q)=\alpha_{k^{\prime}}^{\prime} \tilde{x}_{k^{\prime}}(q)$ for $k, k^{\prime} \in I(y)$, and such that $\|\tilde{x}(q)-y\|=\frac{1}{q}$. Thus, the sequence $\{\tilde{x}(q)\}$ converges to $y$. Suppose that a point $\tilde{x} \in \Re_{+}^{m}$ with the given properties does not exist; then form a sequence $\{x(q)\}$, such that for each integer $q$, $x(q) \in f^{u_{n}^{\prime}}\left(U^{n-1}\right)$, and $x(q) \geq \tilde{x}(q)$. Since the sequence $\{x(q)\}$ is bounded (recall that $X$ is compact), a subsequence converges to some point $x$. Lemma 


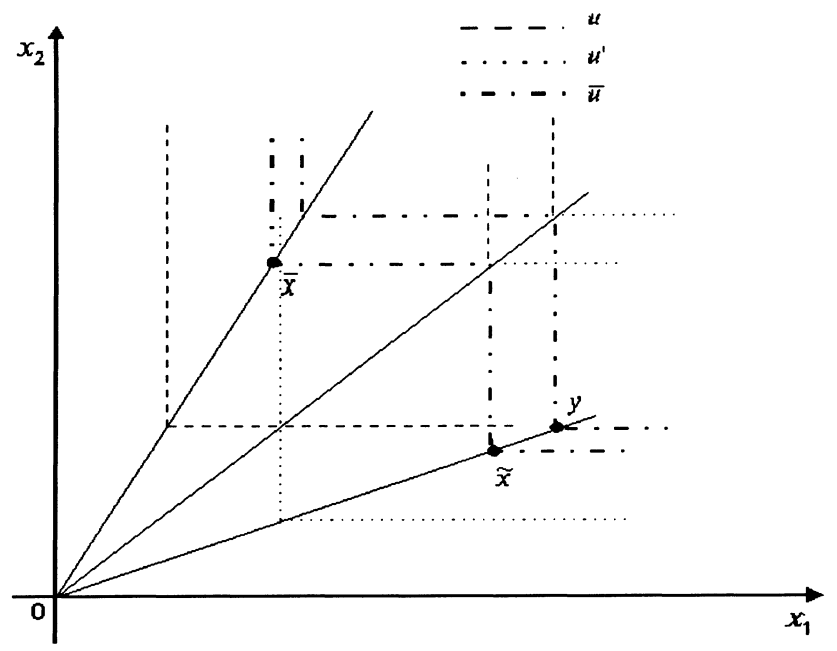

Figure 1

5 implies that there is $x^{\prime} \in f^{u_{n}^{\prime}}\left(U^{n-1}\right)$ such that $x^{\prime} \geq x$. Moreover, since $x(q) \geq \tilde{x}(q)$, then $x^{\prime} \geq x \geq y$. Since $y \in f\left(\widehat{U^{n}}\right)$, and $f^{u_{n}^{\prime}}\left(U^{n-1}\right) \subset f\left(U^{n}\right)$, one has $x^{\prime}=y$. Hence $y \in f^{u_{n}^{\prime}}\left(U^{n-1}\right)$, and therefore $y \in O\left(u_{n}^{\prime}\right)$, which is a contradiction. Thus, a point $\tilde{x}$ with the given properties exists.

Now let $\beta>0$ be such that $u(\bar{x})=\beta u^{\prime}(\tilde{x})$, and consider the utility function $\bar{u} \in U^{*}$ given, for each $x \in \Re_{+}^{m}$, by $\bar{u}(x)=\max \left\{u(x), \beta u^{\prime}(x)\right\}$ (see Figure 1). The function $\bar{u}$ satisfies $m(\bar{u})=y$, and $\bar{u}(\bar{x})>\bar{u}(x)$, for each $x \in f^{u_{n}^{\prime}}\left(U^{n-1}\right) \backslash\{\bar{x}\}$. Thus, because $\bar{x} \in O\left(u_{n}^{\prime}\right)$ by Lemma 3, Lemma 2 implies that $f^{u_{n}^{\prime}}(\bar{u}, \ldots, \bar{u})=$ $f\left(\bar{u}, \ldots, \bar{u}, u_{n}^{\prime}\right)=\bar{x}$. Also as $y \in O\left(u_{n}\right)$, Lemma 2 implies $f^{u_{n}}(\bar{u}, \ldots, \bar{u})=$ $f\left(\bar{u}, \ldots, \bar{u}, u_{n}\right)=y$. Hence

$$
u_{n}\left(f\left(\bar{u}, \ldots, \bar{u}, u_{n}^{\prime}\right)\right)=u_{n}(\bar{x})>u_{n}\left(f\left(\bar{u}, \ldots, \bar{u}, u_{n}\right)\right),
$$

and therefore $f$ is manipulable by Individual $n$ at $\left(\bar{u}, \ldots, \bar{u}, u_{n}\right)$. This contradiction establishes Lemma 8.

Lemma 9 establishes that the correspondence $O$ takes only two possible values on $\dot{U}^{*}$ : it can either be the maximizer of Individual $n$ 's reported utility function, or the entire undominated range.

Lemma 9. For each $u_{n} \in \dot{U}^{*}$, either $O\left(u_{n}\right)=\left\{m\left(u_{n}\right)\right\}$ or $O\left(u_{n}\right)=\widehat{f\left(U^{n}\right)}$.

Proof. Suppose not; let $u_{n} \in \dot{U}^{*}, y \in O\left(u_{n}\right) \backslash\left\{m\left(u_{n}\right)\right\}$, and $z \in \widehat{f\left(U^{n}\right)} \backslash O\left(u_{n}\right)$. By Lemma 8 it can be assumed, w.l.o.g., that $u_{n}(z)>u_{n}(y)$.

Let $u \in U^{*}$ be such that $m(u)=z$, and such that $y$ uniquely maximizes $u$ on $f^{u_{n}}\left(U^{n-1}\right)$ (a function with these features can be constructed as in the proof of Lemma 8). Lemma 2 yields $f^{u_{n}}(u, \ldots, u)=f\left(u, \ldots, u, u_{n}\right)=y$. Also Lemma 2 implies $f(u, \ldots, u, u)=z$. Then 


$$
u_{n}(f(u, \ldots, u, u))=u_{n}(z)>u_{n}(y)=u_{n}\left(f\left(u, \ldots, u, u_{n}\right)\right),
$$

and therefore $f$ is manipulable by Individual $n$ at $\left(u, \ldots, u, u_{n}\right)$. This contradiction establishes Lemma 9.

Lemma 10 establishes, for strategy-proof mechanisms satisfying the assumptions of Theorem 1, that the correspondence $O$ is constant on $\dot{U}^{*}$.

Lemma 10. Assume that $\widehat{f\left(U^{n}\right)}$ contains three or more outcomes. If there is $u_{n} \in \dot{U}^{*}$ such that $O\left(u_{n}\right)=\left\{m\left(u_{n}\right)\right\}$, then for each $u_{n} \in \dot{U}^{*}$, one has $O\left(u_{n}\right)=\left\{m\left(u_{n}\right)\right\}$.

Proof. Suppose not; let $u_{n}, u_{n}^{\prime} \in \dot{U}^{*}$ be such $O\left(u_{n}\right)=\left\{m\left(u_{n}\right)\right\}$ and $O\left(u_{n}^{\prime}\right)=\widehat{f\left(U^{n}\right)}$ (Lemma 9). Choose $\bar{x} \in f\left(\widehat{U^{n}}\right) \backslash\left\{m\left(u_{n}\right), m\left(u_{n}^{\prime}\right)\right\}$; by Lemma 8 it can assumed, w.l.o.g., that $u_{n}^{\prime}\left(m\left(u_{n}\right)\right)>u_{n}^{\prime}(\bar{x})$. Let $\bar{u} \in U^{*}$ be such that $m(\bar{u})=\bar{x}$, and consider that sequence $\left\{f\left(\bar{u}^{q}, \ldots, \bar{u}^{q}, u_{n}^{\prime}\right)\right\}$, where $\bar{u}^{q}(x)=\bar{u}(x)+1 / q \sum_{k 1}^{m} x_{k}$. Since $\left(\bar{u}^{q}, \ldots, \bar{u}^{q}, u_{n}^{\prime}\right) \in \dot{U}^{n}$, then $f\left(\bar{u}^{q}, \ldots, \bar{u}^{q}, u_{n}^{\prime}\right)$ $\in O\left(u_{n}^{\prime}\right)$, and therefore one of its subsequences, $\left\{f\left(\bar{u}^{q_{s}}, \ldots, \bar{u}^{q_{s}}, u_{n}^{\prime}\right)\right\}$, converges to $\bar{x}$. Also $\left\{f\left(\bar{u}^{q}, \ldots, \bar{u}^{q}, u_{n}\right)\right\} \subset O\left(u_{n}\right)=\left\{m\left(u_{n}\right)\right\}$; i.e., $f\left(\bar{u}^{q}, \ldots, \bar{u}^{q}, u_{n}\right)$ $=m\left(u_{n}\right)$ for each $q$. Since $f$ is strategy-proof one has

$$
u_{n}^{\prime}\left(f\left(\bar{u}^{q_{s}}, \ldots, \bar{u}^{q_{s}}, u_{n}^{\prime}\right)\right) \geq u_{n}^{\prime}\left(f\left(\bar{u}^{q_{s}}, \ldots, \bar{u}^{q_{s}}, u_{n}\right)\right)=u_{n}^{\prime}\left(m\left(u_{n}\right)\right),
$$

for each $q_{s}$, and therefore in the limit $u_{n}^{\prime}(\bar{x}) \geq u_{n}^{\prime}\left(m\left(u_{n}\right)\right)$. This contradiction establishes Lemma 10.

Proof of Theorem 1: Let $f$ be a mechanism satisfying the assumptions of Theorem 1. First, it is shown by induction on the number of individuals that $f$ is dictatorial on $\left(\dot{U}^{*}\right)^{n}$. When $n=1$, this is an implication of Lemma 2. Assume that this claim is true for every mechanism for which $n \leq K-1$. It is shown that the claim holds for $n=K$.

By Lemma 10, either $O\left(u_{n}\right)=\left\{m\left(u_{n}\right)\right\}$ for each $u_{n} \in \dot{U}^{*}$, or $O\left(u_{n}\right)=\widehat{f\left(U^{n}\right)}$ for each $u_{n} \in \dot{U}^{*}$. If $O\left(u_{n}\right)=\left\{m\left(u_{n}\right)\right\}$ for each $u_{n} \in \dot{U}^{*}$, then $f(\mathbf{u})=m\left(u_{n}\right)$ for each $\mathbf{u} \in\left(\dot{U}^{*}\right)^{n}$, and therefore Individual $n$ is a dictator for $f$ on $\left(\dot{U}^{*}\right)^{n}$. If $O\left(u_{n}\right)=\widehat{f\left(U^{n}\right)}$ for each $u_{n} \in \dot{U}^{*}$, then consider the mechanism $f^{u_{n}}: U^{n-1} \rightarrow X$ given for each $\mathbf{u}_{-n} \in U^{n-1}$ by $f^{u_{n}}\left(\mathbf{u}_{-n}\right)=f\left(\mathbf{u}_{-n}, u_{n}\right)$. Each $f^{u_{n}}$ is a strategy-proof mechanism satisfying the assumptions of Theorem 1. (It is easy to see that $\left.f^{u_{n}} \widehat{\left(U^{n}-1\right.}\right)=\widehat{f\left(U^{n}\right)}$ : let $x \in \widehat{f\left(U^{n}\right)}$; because $f^{u_{n}}\left(U^{n-1}\right)$ $\subset f\left(U^{n}\right)$, and $f^{u_{n}} \widehat{\left(U^{n}-1\right)} \supset \widehat{f\left(U^{n}\right)}$, then $x \in f^{u_{n}} \widehat{\left(U^{n}-1\right)}$. Let $x \in f\left(U^{n}\right) \backslash \widehat{f\left(U^{n}\right)}$; then by Lemma 7 there is $x^{\prime} \in f\left(\widehat{\left.U^{n}\right)} \subset f^{u_{n}} \widehat{\left(U^{n}-1\right)}\right.$ such that $x^{\prime}>x$; hence $x \notin f^{u_{n}} \widehat{\left(U^{n}-1\right)}$.) Thus the induction hypothesis implies that each $f^{u_{n}}$ is dictatorial on $\left(\dot{U}^{*}\right)^{n-1}$. It is shown that a single Individual (always the same) is the dictator for each $f^{u_{n}}$ on $\left(\dot{U}^{*}\right)^{n-1}$.

Suppose not; w.l.o.g., assume that Individual 1 is the dictator for $f^{u_{n}}$ on $\left(\dot{U}^{*}\right)^{n-1}$, and Individual 2 is the dictator for $f^{u_{n}^{\prime}}$ on $\left(\dot{U}^{*}\right)^{n-1}$. Let $\mathbf{u}_{-\{1,2, n\}}$ $\in\left(\dot{U}^{*}\right)^{n-3}$ arbitrary, and let $u_{1}, u_{2} \in \dot{U}^{*}$ be such that $m\left(u_{1}\right) \neq m\left(u_{n}\right)$, and $m\left(u_{2}\right)=m\left(u_{n}\right)$. Hence $f^{u_{n}}\left(u_{1}, u_{2}, \mathbf{u}_{-\{1,2, n\}}\right)=f\left(u_{1}, u_{2}, \mathbf{u}_{-\{1,2, n\}}, u_{n}\right) \neq m\left(u_{n}\right)$, 
and $f^{u_{n}^{\prime}}\left(u_{1}, u_{2}, \mathbf{u}_{-\{1,2, n\}}\right)=f\left(u_{1}, u_{2}, \mathbf{u}_{-\{1,2, n\}}, u_{n}^{\prime}\right)=m\left(u_{n}\right)$, and therefore $f$ is manipulable by Individual $n$ at $\left(u_{1}, u_{2}, \mathbf{u}_{-\{1,2, n\}}, u_{n}\right)$. This contradiction establishes that some Individual $i$ is the dictator for each $f^{u_{n}}$ on $\left(\dot{U}^{*}\right)^{n-1}$ (i.e., for each $\left.\mathbf{u} \in\left(\dot{U}^{*}\right)^{n}, f^{u_{n}}\left(\mathbf{u}_{-n}\right)=f(\mathbf{u})=m\left(u_{i}\right)\right)$, thereby showing that $f$ is dictatorial on $\left(\dot{U}^{*}\right)^{n}$.

Assume, w.l.o.g., that Individual 1 is the dictator for $f$ on $\left(\dot{U}^{*}\right)^{n}$. It is shown that Individual 1 is the dictator for $f$ on $\dot{U}^{*} \times \dot{U}^{n-1}$. Suppose not; let $\mathbf{u} \in \dot{U}^{*} \times \dot{U}^{n-1}$ be such that $f(\mathbf{u}) \neq m\left(u_{1}\right)$. Then let $\bar{u} \in \dot{U}^{*}$ be such that $\bar{u}(f(\mathbf{u}))>\bar{u}\left(m\left(u_{1}\right)\right)$. (A function with this feature can be constructed as follows: Write $f(\mathbf{u})=\bar{x}$. Let $\alpha \in \Re^{m}, \alpha \gg 0$, be such that $\alpha_{k} \bar{x}_{k}=\alpha_{k^{\prime}} \bar{x}_{k^{\prime}}$ for each $k \in I(\bar{x})$, and let $u$ be given for each $x \in \Re_{+}^{m}$ by $u(x)=$ $\min \left\{\alpha_{k} x_{k}, k \in I(\bar{x})\right\}$; let $\epsilon>0$ be sufficiently small that for each $x \in f\left(U^{n}\right)$, $\|x-\bar{x}\|<\epsilon$ implies that $u(x)>u\left(m\left(u_{1}\right)\right)$, and let $\bar{x}^{\prime}<\bar{x}$ be such that $\alpha_{k} \bar{x}_{k}^{\prime}=$ $\alpha_{k^{\prime}} \bar{x}_{k^{\prime}}^{\prime}$ and $\left\|\bar{x}^{\prime}-\bar{x}\right\|<\epsilon$. Define $u^{q}(x)=u(x)+\frac{1}{q} \sum_{k}^{n} x_{k} x_{k}$, for each $x \in \Re_{+}^{m}$, and let $\bar{q}$ be an integer sufficiently large that for each $x \in f\left(U^{n}\right), u^{\bar{q}}(x) \geq u^{\bar{q}}(\bar{x})$ implies $x>\bar{x}^{\prime}$; such integer $\bar{q}$ exists because $f\left(U^{n}\right) \subset X$, and $X$ is a compact set. Denote by $M\left(u^{\bar{q}}\right)$ the set of maximizers of $u^{\bar{q}}$ on $f\left(U^{n}\right) ; M\left(u^{\bar{q}}\right)$ is nonempty by Lemma 2. Finally, let $\hat{x} \in M\left(u^{\bar{q}}\right)$; note that $\hat{x} \in f\left(\widehat{\left.U^{n}\right)}\right.$ as $u^{\bar{q}}$ is a strictly increasing utility function. By slightly bending $u^{\bar{q}}$ is indifference curve through $\hat{x}$ in the direction of the main diagonal, one can construct a utility function $\bar{u} \in \dot{U}^{*}$ such that $m(\bar{u})=\hat{x}$.) Lemma 2 (applied to $f^{u_{1}}$ ) implies

$$
\bar{u}\left(f\left(u_{1}, \bar{u}, \ldots, \bar{u}\right)\right) \geq \bar{u}(f(\mathbf{u}))>\bar{u}\left(m\left(u_{1}\right)\right) ;
$$

hence $f\left(u_{1}, \bar{u}, \ldots, \bar{u}\right) \neq m\left(u_{1}\right)$. But notice that $\left(u_{1}, \bar{u}, \ldots, \bar{u}\right) \in\left(\dot{U}^{*}\right)^{n}$, and this contradicts that Individual 1 is the dictator for $f$ on $\left(\dot{U}^{*}\right)^{n}$.

In order to establish Theorem 1, it must be shown that for each $\mathbf{u} \in \dot{U}^{n}, f$ maximizes $u_{1}$ on $f\left(U^{n}\right)$. Suppose not; i.e., let $\mathbf{u} \in \dot{U}^{n}$, and $\tilde{x} \in f\left(U^{n}\right)$ be such that $u_{1}(\tilde{x})>u_{1}(f(\mathbf{u}))$. Let $\tilde{u} \in \dot{U}^{*}$ be such that $u_{1}(m(\tilde{u}))>u_{1}(f(\mathbf{u}))$. Since Individual 1 is the dictator for $f$ on $\dot{U}^{*} \times \dot{U}^{n-1}$, one has

$$
u_{1}\left(f\left(\tilde{u}, \mathbf{u}_{-1}\right)\right)=u_{1}(m(\tilde{u}))>u_{1}(f(\mathbf{u})),
$$

and therefore $f$ is manipulable by Individual 1 at $\mathbf{u}$, contradicting that $f$ is strategy-proof and establishing Theorem 1.

Lemma 11 establishes that a strategy-proof mechanism $f$ satisfying the assumptions of Theorem 1 must always select outcomes that are less than or equal to the most preferred bundle of the dictator for $f$ on $\dot{U}^{n}$. This property will be useful in the proof of Proposition 1.

Lemma 11. If $f$ is a mechanism satisfying the assumptions of Theorem 1, then there is $i \in N$ such that for each $\left(u_{i}, \mathbf{u}_{-i}\right) \in U^{*} \times U^{n-1}, f\left(u_{i}, \mathbf{u}_{-i}\right) \leq m\left(u_{i}\right)$.

Proof. Assume, w.l.o.g., that Individual 1 is the dictator for $f$ on $\dot{U}^{n}$ (Theorem 1). It is shown that Lemma 11 holds for $i=1$. Suppose not; let $\left(u_{1}, \mathbf{u}_{-1}\right) \in U^{*} \times U^{n-1}$ and suppose that it is not the case that $f\left(u_{1}, \mathbf{u}_{-1}\right) \leq m\left(u_{1}\right)$. Let $u \in \dot{U}$ be such that $u\left(f\left(u_{1}, \mathbf{u}_{-1}\right)\right)>u\left(m\left(u_{1}\right)\right)$. Since $f\left(u_{1}, \mathbf{u}_{-1}\right) \in f\left(u_{1}, U^{n-1}\right)$, Lemma 2 (applied to $\left.f^{u_{1}}\right)$ yields 


$$
u\left(f\left(u_{1}, u, \ldots, u\right)\right) \geq u\left(f\left(u_{1}, \mathbf{u}_{1}\right)\right) .
$$

Hence $f\left(u_{1}, u, \ldots, u\right) \neq m\left(u_{1}\right)$.

Let $\bar{q}$ be a sufficiently large integer that $u_{1}^{\bar{q}}(x)=u_{1}(x)+\frac{1}{\bar{q}} \sum_{k}^{m} x_{k} x_{k}$ satisfies that for each $x \in f\left(U^{n}\right), u_{1}^{\bar{q}}(x) \geq u_{1}^{\bar{q}}\left(m\left(u_{1}\right)\right)$ implies $u_{1}(x)>u_{1}\left(f\left(u_{1}, u, \ldots, u\right)\right)$ (recall that $X \supset f\left(U^{n}\right)$ is a compact set). Since $\left(u_{1}^{\bar{q}}, u, \ldots, u\right) \in \dot{U}^{n}$, and Individual 1 is the dictator for $f$ on $\dot{U}^{n}$, then one has $u_{1}^{\bar{q}}\left(f\left(u_{1}^{\bar{q}}, u, \ldots, u\right)\right) \geq$ $u_{1}^{\bar{q}}\left(m\left(u_{1}\right)\right)$. Hence

$$
u_{1}\left(f\left(u_{1}^{\bar{q}}, u, \ldots, u\right)\right)>u_{1}\left(f\left(u_{1}, u, \ldots, u\right)\right),
$$

and therefore $f$ is manipulable by Individual 1 at $\left(u_{1}, u, \ldots, u\right)$, which contradicts that $f$ is strategy-proof and proves the lemma.

Now Proposition 1 can be easily proved.

Proof of Proposition 1. Assume, w.l.o.g., that Lemma 11 is satisfied for $i=1$. It is shown that Individual 1 is a dictator for $f$. Suppose not; let $\mathbf{u} \in U^{n}$ and $x \in f\left(U^{n}\right)$ be such that $u_{1}(x)>u_{1}(f(\mathbf{u}))$. By Lemma 7 it can be assumed w.l.o.g. that $x \in \widehat{f\left(U^{n}\right)}$. Hence let $u_{1}^{\prime} \in U^{*}$ be such that $m\left(u_{1}^{\prime}\right)=x$. Lemma 11 yields $f\left(u_{1}^{\prime}, \mathbf{u}_{1}\right) \leq x$; moreover, $f\left(u_{1}^{\prime}, \mathbf{u}_{1}\right) \in \widehat{f\left(U^{n}\right)}$ as $f$ is non-wasteful. Hence $f\left(u_{1}^{\prime}, \mathbf{u}_{1}\right)=x$. This implies, however, that $f$ is manipulable by Individual 1 at $\mathbf{u}$, contradicting that $f$ is strategy-proof. Hence Individual 1 is a dictator for $f$ on $U^{n}$, and therefore $f$ is dictatorial.

Proof of Proposition 2. Assume that $\dot{U}$ is the set of all the continuous strictly increasing utility functions, and let $f: \dot{U}^{n} \rightarrow X$ be a strategy-proof mechanism such that $\overline{f\left(\dot{U}^{n}\right)}$ contains three or more outcomes. Write $U$ for the set of all the continuous increasing utility functions. Proposition 2 is proved by showing that $f$ can be extended to a mechanism $F: U^{n} \rightarrow X$ satisfying the assumptions of Theorem 1. The mechanism $F$ will therefore be dictatorial on $\dot{U}^{n}$ by Theorem 1 , and as $F$ coincides with $f$ on $\dot{U}^{n}$, then $f$ is (fully) dictatorial.

The mechanism $F \equiv F^{n}$ is defined recursively as follows: $F^{0} \equiv f$, and $F^{i}: U^{i} \times \dot{U}^{n} i \rightarrow X$, is given by

$$
F^{i}(\mathbf{u})=\left\{\begin{array}{cc}
F^{i 1}(\mathbf{u}) & \text { if } \mathbf{u} \in U^{i 1} \times \dot{U}^{n} i+1 \\
\sigma\left(M_{i}(\mathbf{u})\right) & \text { otherwise }
\end{array}\right.
$$

where $\sigma$ is an arbitrary selection on $X$, and $M_{i}(\mathbf{u}) \equiv M_{i}^{n}(\mathbf{u})$ is defined by letting $M_{i}^{0}(\mathbf{u})$ be the set of maximizers of $u_{i}$ on $\overline{F^{i} 1\left(\dot{U}, \mathbf{u}{ }_{i}\right)}$ (a nonempty compact set), and $M_{i}^{j}(\mathbf{u})$ be the set of maximizers of $u_{j}$ on $M_{i}^{j}{ }^{1}(\mathbf{u})$, for $j=1, \ldots, n$. Note that for each $\mathbf{u} \in \dot{U}^{n}, F(\mathbf{u})=f(\mathbf{u})$.

In order to show that $F$ is strategy-proof, it is shown by induction that every mechanism $F^{0}, F^{1}, \ldots, F^{n}$ is strategy-proof. Clearly $F^{0}$ is strategyproof. Assuming that $F^{0}, \ldots, F^{i}{ }^{1}$ are strategy-proof, we must show that $F^{i}$ is strategy-proof also. 
It is easy to show that $F^{i}$ is not manipulable by Individual $i$, for if there is $\mathbf{u} \in U^{i} \times \dot{U}^{n}{ }^{i}$ and $u_{i}^{\prime} \in U$ such that $u_{i}\left(F^{i}(\mathbf{u})\right)<u_{i}\left(F^{i}\left(u_{i}^{\prime}, \mathbf{u}{ }_{i}\right)\right)$, then because $u_{i}$ is continuous and $F^{i}\left(u_{i}^{\prime}, \mathbf{u}{ }_{i}\right) \in \overline{F^{i}{ }^{1}\left(\dot{U}, \mathbf{u}_{i}\right)}$, there is $\bar{u}_{i}^{\prime} \in \dot{U}$ such that $F^{i}{ }^{1}\left(\bar{u}_{i}^{\prime}, \mathbf{u}_{i}\right)$ is sufficiently close to $F^{i}\left(u_{i}^{\prime}, \mathbf{u}_{i}\right)$ that $u_{i}\left(F^{i}(\mathbf{u})\right)<u_{i}$ $\left(F^{i} 1\left(\bar{u}_{i}^{\prime}, \mathbf{u}_{i}\right)\right)$; this contradicts that $F^{i}(\mathbf{u})$ is a maximizer of $u_{i}$ on $\overline{F^{i} 1} 1\left(\dot{U}, \mathbf{u}_{i}\right)$.

Suppose by way of contradiction that Individual $j \in N \backslash\{i\}$ can manipulate $F^{i}$ at $\mathbf{u}$; i.e., there is an admissible $u_{j}^{\prime}$ such that $u_{j}\left(F^{i}(\mathbf{u})\right)<u_{j}\left(F^{i}\right.$ $\left.\left(\mathbf{u}_{j}, u_{j}^{\prime}\right)\right)$. Write $F^{i}\left(\mathbf{u}_{j}, u_{j}^{\prime}\right)=x, F^{i}(\mathbf{u})=y, \overline{F^{i}{ }^{1}\left(\dot{U}, \mathbf{u}_{\{i, j\}}, u_{j}\right)}=\bar{O}\left(u_{j}\right)$, and $\overline{F^{i}{ }^{1}\left(\dot{U}, \mathbf{u}\{i, j\}, u_{j}^{\prime}\right)}=\bar{O}\left(u_{j}^{\prime}\right)$. By the definition of $F^{i}$, we have $x \notin M_{i}^{j}(\mathbf{u})$. Suppose that $x \in \bar{O}\left(u_{j}\right)$; then there is $\bar{j} \in\{1, \ldots, j-1\} \cup\{i\}$ such that $u_{\bar{j}}(y)>u_{\bar{j}}(x)$, and $u_{j^{\prime}}(y)=u_{j^{\prime}}(x)$ for $j^{\prime} \in\{1, \ldots, \bar{j}-1\} \cup\{i\}$. As $F^{i}\left(\mathbf{u}_{j}, u_{j}^{\prime}\right)$ $=x$, then $y \notin M_{i}^{j}\left(\mathbf{u}_{j}, u_{j}^{\prime}\right)$, and as $u_{j^{\prime}}(y)=u_{j^{\prime}}(x)$ for $j^{\prime} \in\{1, \ldots, \bar{j}-1\} \cup\{i\}$, one must have $y \notin \bar{O}\left(u_{j}^{\prime}\right)$. Therefore either

(2.1) $y \notin \bar{O}\left(u_{j}^{\prime}\right)$, or

(2.2) $x \notin \bar{O}\left(u_{j}\right)$.

We show that (2.1) implies that $F^{i} 1$ is manipulable by Individual $j$, leading to a contradiction as $F^{i} 1$ is strategy proof by the induction hypothesis. By simply commuting the roles of $x$ and $y$, and $\bar{O}\left(u_{j}^{\prime}\right)$ and $\bar{O}\left(u_{j}\right)$ in the definition of $\bar{u}$ given below, an identical argument shows that (2.2) also leads to a contradiction.

Let $\bar{u} \in U$ be such that $y$ is its unique maximizer on $\overline{f\left(\dot{U}^{n}\right)}$, and such that $\bar{u}(x)>\bar{u}\left(x^{\prime}\right)$, for each $x^{\prime} \in \bar{O}\left(u_{j}^{\prime}\right) \backslash\{x\}$. (As $f\left(\dot{U}^{n}\right) \subset f\left(\widehat{\dot{U}^{n}}\right)$ by Lemma 4, a utility function with these features can be constructed as in the proof Lemma 8 under (1.1).) For each integer $q$ define the utility function $u^{q}$ by $u^{q}(x)=\bar{u}(x)+\frac{1}{q} \sum_{k}^{m}{ }_{1} x_{k}$, for $x \in \Re_{+}^{m}$. The sequence $\left\{F^{i}{ }^{1}\left(\bar{u}^{q}, \mathbf{u}\{i, j\}, u_{j}^{\prime}\right)\right\}$ $\subset \bar{O}\left(u_{j}^{\prime}\right)$ has a convergent subsequence, $\left\{F^{i}{ }^{1}\left(\bar{u}^{q_{s}}, \mathbf{u}\{i, j\}, u_{j}^{\prime}\right)\right\}$; because $F^{i}{ }^{1}$ is strategy-proof, the limit of this subsequence can only be $x$. Also the sequence $\left\{F^{i}{ }^{1}\left(\bar{u}^{q_{s}}, \mathbf{u}_{i}\right)\right\} \subset \bar{O}\left(u_{j}\right)$ or one of its subsequences converges to $y$. As $F^{i}{ }^{1}$ is strategy-proof, for each integer $q_{s}$ one has

$$
u_{j}\left(F^{i}{ }^{1}\left(\bar{u}^{q_{s}}, \mathbf{u}_{i}\right)\right) \geq u_{j}\left(F^{i}{ }^{1}\left(\bar{u}^{q_{s}}, \mathbf{u}_{\{i, j\}}, u_{j}^{\prime}\right)\right),
$$

and therefore in the limit $u_{j}(y) \geq u_{j}(x)$, which is a contradiction.

Thus, $F$ is strategy-proof. Moreover, by the definition of $F$ and Lemma 2, $F\left(U^{n}\right)=\overline{f\left(\dot{U}^{n}\right)}$, and therefore $F$ satisfies the assumptions of Theorem 1 .

\section{References}

1. Barberà, S., Jackson, M.: A characterization of strategy-proof voting social choice functions for economies with pure public goods. Social Choice and Welfare 11, 241252 (1994)

2. Barberà, S., Peleg, B.: Strategy-proof voting schemes with continuous preferences. Social Choice and Welfare 7, 3138 (1990) 
3. Gibbard, A.: Manipulation of voting schemes: a general result. Econometrica 41, 587-602 (1973)

4. Hildenbrand, W.: Core and equilibria of a large economy. Princeton, New Jersey: Princeton University Press 1974

5. Mas-Colell, A.: Continuous and Smoth consumers approximation theorems, Journal of Economic Theory 8, 305-336 (1974)

6. Moreno, D.: Nonmanipulable decision mechanisms for economic environments. Social Choice and Welfare 11, 225-240 (1994)

7. Moreno, D.: Strategy-proof mechanisms with monotonic preferences: the case of pure public goods economies. Universidad Carlos III, WP 94-24 (1995)

8. Moulin, H.: On strategy-proofness and single peakedness, Public Choice 35, 437-455 (1980)

9. Munkres, J. R.: Topology, a first course. New York: Prentice Hall 1987

10. Satterthwaite, M.: Strategy-proofness and Arrow's conditions: existence and correspondence theorems for voting procedures and social welfare functions. Journal of Economic Theory 35, 1-18 (1975)

11. Zhou, L.: Impossibility of strategy-proof allocation mechanisms in economies with public goods. Review of Economic Studies 58, 107-119 (1991)

12. Zhou, L.: Inefficiency of strategy-proof allocation mechanisms in pure exchange economies. Social Choice and Welfare 8, 247-254 (1991) 